\title{
LA HUMANIDAD EN EL LIMBO. EL SISTEMA DE PROTECGIÓN INTERNACIONAL DE TURQUÍA ANTE LA CRISIS HUMANITARIA DEL CONFLICTO EN SIRIA
}

\author{
HUMANITY IN LIMBO. \\ TURKEY'S INTERNATIONAL PROTECTION SYSTEM \\ IN THE FACE OF THE HUMANITARIAN CRISIS \\ ARISING FROM THE CONFLICT IN SYRIA
}

\section{L'HUMANITÉ DANS LES LIMBES. LE SYSTÉME DE PROTECTION INTERNATIONALE DE LA TURQUIE FACE À LA CRISE HUMANITAIRE DÉCOULANT DU CONFLIT EN SYRIE}

\author{
Verónica S. Souto Olmedo \\ Instituto Tecnológico y de Estudios Superiores de Occidente \\ veronica.souto.olmedo@gmail.com
}

Resumen: El conflicto sirio ha provocado una catástrofe humanitaria con 6.1 millones de desplazados internos y más de cinco de refugiados. Turquía es un actor fundamental en el conflicto por su involucramiento militar y por albergar a 3.5 millones de refugiados sirios registrados. El presente artículo se concentrará en la crisis humanitaria y buscará comprobar la relación entre el sistema de protección internacional turco y la estrategia para gestionar tal crisis, la cual se ha basado en dos premisas: por una parte, la estancia temporal de los refugiados y, por otra, la provisión de servicios, antes bien que en el reconocimiento de derechos.

Palabras clave: Turquía, Siria, refugiados, protección temporal, ACNUR.

Abstract: The Syrian conflict has caused a humanitarian catastrophe with 6.1 million internally displaced persons and more than 5 million refugees. Turkey is a key player in the conflict because of its military involvement and because it hosts 3.5 million registered Syrian refugees. This paper focuses on the humanitarian crisis and seeks to verify the relationship between the Turkish international protection system and the strategy to manage this crisis, which has 
been based on two premises: on the one hand, the temporary character of the refugees and, on the other hand, the provision of services, rather than the recognition of rights.

Keywords: Turkey, Syria, refugees, temporary protection, UNHCR.

Traducción de Gonzalo Celorio Morayta

Résumé: Le conflit syrien a provoqué une catastrophe humanitaire, en raison des 6.1 millions de déplacés à l'intérieur du pays, auxquels s'ajoutent cinq millions de réfugiés. La Turquie est un acteur fondamental dans ce conflit, à cause de sa participation militaire et parce qu'elle a reçu trois millions et demi de réfugiés syriens enregistrés. L'article est centré sur la crise humanitaire et cherche à prouver un rapport entre le système de protection internationale turc et la stratégie pour gérer la crise. Celle-ci est fondée sur deux prémisses: que la présence des réfugiés est temporaire et qu'il faut leur procurer des services, plutôt que de leur accorder des droits.

Mots clefs: Turquie, Syrie, réfugiés, protection temporaire, HCR.

Traducción de BERNARdo Mabire

Fecha de recepción: enero 2018 Fecha de aceptación: mayo de 2018 
$\mathrm{E}$ N DICIEMBRE DE 2010, el mundo fue testigo de un movimiento tectónico en los regímenes políticos del Medio Oriente, conocido globalmente como Primavera Árabe. El conflicto sirio, prolongado y complejizado por la irrupción de Dáesh (al-Dawla al-Islāmīya fī al-Irā $q$ wa-al-Shām $)^{1}$ desde Iraq, a más de la intervención de potencias regionales y globales, ha suscitado una de las mayores crisis humanitarias de las últimas décadas.

Si bien los números no alcanzan a describir el drama humano, nos dan una idea del estado de la situación. El conflicto ha causado, según datos del Alto Comisionado de Naciones Unidas para los Refugiados (ACNUR), 6.1 millones de personas internamente desplazadas (2.8 millones de niños), hacia diciembre de 2017, y más de cinco millones (5615147) de refugiados, hacia el 15 de marzo de $2018,{ }^{2}$ mayoritariamente situados en el Líbano (991917), Jordania (659 063) y Turquía (3554496). ${ }^{3}$ Es difícil obtener una cifra exacta de víctimas

${ }^{1}$ Véase T. Singer, "3Qs: What using the name «Daesh", rather than «ISIS» or «ISIL» Really Means", News@Northeastern, en http://news.northeastern.edu/2015/11/3qs-what-using-the-name-daesh-rather-than-isisor-isil-really-means, consultado el 30.IV.2017. El acrónimo Dáesh se utiliza en el mundo islámico para nombrar de manera peyorativa al grupo terrorista conocido comúnmente como Estado Islámico de Iraq y el Levante (ISIL, por sus siglas en inglés) o Estado Islámico de Iraq y Siria (ISIs, por sus siglas en inglés), toda vez que da-ish, en árabe, tiene connotaciones negativas, como 'aquel que comete crímenes aborrecibles', 'aquel que aplasta'. Por lo demás, Dáesh no alude a las palabras "Estado" o "islámico", por lo cual el mundo árabe en particular no reconoce ninguna de estas características en el citado grupo terrorista.

${ }^{2}$ ACNUR, "Syria Regional Refugee Response. Inter-agency Information Sharing Portal", en http://data.unhcr.org/syrianrefugees/regional. php, consultado el 21.III.2018.

${ }^{3}$ Según cifras de la Dirección General de Manejo de la Migración (DGMM), al 15 de marzo de 2018, de los 3554496 de refugiados sirios, 1.8 se encuentra en la zona fronteriza con Siria. Del total de refugiados, menos del diez por ciento, unos 227802 están en 21 campos en el sur del país y el resto, disperso por todo el territorio. Los sirios reasentados en la UE según el Acuerdo de 2016 han sido 12 640. Véase DGMM, "Temporary Protection Statistics", en http://www.goc.gov.tr/icerik6/temporary-protection_915_1024_4748_icerik, consultado el 21.III.2018. 
fatales, ya que éstas difieren sustancialmente según la fuente, pero el dato oficial más reciente indica que, en abril de 2016, el conflicto había cobrado la vida de 400 mil personas, ${ }^{4}$ sin contar a quienes perecieron ni en el viaje a Europa ni durante los años posteriores.

Tras estallar el conflicto en Siria, el primer grupo de refugiados ingresó en Hatay (Turquía), en abril de 2011. En ese momento, el gobierno adoptó una política de "puertas abiertas" para recibir a todos aquellos que huían del conflicto. Esta decisión se basó en el supuesto según el cual Bashar alAssad iba a caer pronto y los refugiados iban a regresar a sus hogares. Se pensaba, por lo demás, que tal acción haría de Turquía un modelo democrático para el Medio Oriente, al reforzar la política neotomana que venía implementando Ankara. El entonces primer ministro, Recep Tayyip Erdoğan, afirmó que pronto estaría en Damasco y que recitaría la AlFatiha tanto en la tumba de Saladino, cuanto en los patios de la Gran Mezquita de los Omeyas. En consecuencia, el ministro de Relaciones Exteriores turco, Ahmet Davutoğlu, tasó la recepción de refugiados para el país en 100 mil.

La realidad y el tiempo han refutado estas aseveraciones y actualmente Turquía alberga unos 3.5 millones de refugiados sirios registrados hacia marzo de 2018, que se suman a los 330500 refugiados no sirios registrados en el ACNUR provenientes mayormente de Iraq, Afganistán e Irán (según datos hasta el 30 de septiembre de 2017). ${ }^{5}$ Decir no está de más que numerosas organizaciones no gubernamentales (ONG) cifran el número total en cuatro millones por la cantidad de personas que deciden no registrarse por temor a ser discriminadas (minorías religiosas y étnicas) o para no ser detectadas

${ }^{4}$ Declaración de Staffan de Mistura, Enviado de las Naciones Unidas y la Liga Árabe para Siria, apud J. Hudson, "U.N. Envoy Revises Syria Death Toll to 400,000", Foreign Policy, 22 de abril de 2016, en http://foreignpoli cy.com/2016/04/22/u-n-envoy-revises-syria-death-toll-to-400000/, consultado el 28.XII.2017.

${ }^{5}$ ACNUR, "UNHCr Turkey's Monthly Statistics", en https://data2.unhcr.org/fr/documents/download/57101, consultado el 29.XII.2017. 
en su camino hacia Europa. ${ }^{6}$ De otra parte, algunas ciudades albergan un porcentaje significativo de refugiados, entre las cuales descuellan Kilis (95.48 \%), Hatay (28.46 \%), Şanlıurfa $(24.05 \%)$ y Gaziantep $(18.64 \%) .^{7}$

El derecho de los refugiados, en estas circunstancias, cobra una relevancia capital para proteger a las personas que huyen de conflictos armados a fin de asegurarles sus derechos fundamentales. Oktay Durukan explica que el derecho de los refugiados es una excepción al principio de soberanía y que los estados deben abrir sus puertas a los perseguidos, porque "los refugiados de un estado son responsabilidad de todos". 8 Sin embargo, como situación de excepción, tiene numerosas ambigüedades, continúa Durukan, y da mucho espacio de maniobra a los gobiernos. Esa libertad les permite condicionar el otorgamiento, alcance e implementación de la protección internacional sin violar el derecho internacional.

Este artículo, por cuanto se ha apuntado líneas arriba, intentará comprobar que hay una relación entre el sistema de protección internacional turco y la estrategia de Ankara para gestionar la crisis humanitaria, que fue catalogada como una situación de emergencia transitoria y se ha basado en dos pilares: la estancia temporal de los refugiados, por una parte,

${ }^{6}$ Según un estudio de M. Erdoğan y C. Ünver (Perspectives, Expectations and Suggestions of the Turkish Business Sector: Syrians in Turkey, Ankara, Turkish Confederation of Employer Associations, 2015), algunas autoridades locales también manejan cifras más altas a las publicadas: en Şanliurfa cifraban el número en 500 mil (frente a 365 mil registrados); en Estambul calculaban 400 mil (frente a 305 mil registrados). Hay varias razones por las que las personas no se registran: aquellos bajo protección temporal no pueden solicitar residencia en un tercer país, los solicitantes no pueden mudarse de la provincia de registro, y algunos ven que el régimen sirio podría considerar el registro un auténtico problema. Los autores citan otras dos causas importantes: los primeros dos años no hubo un registro centralizado y confiable, y las personas que parten ilegalmente a un tercer país no cancelan su registro.

7 DGMM, "Temporary Protection Statistics".

8 "Who are the Refugees, What do They Teach Us?", Saha. Citizen's Assembly Turkey, núm. esp. 1, 2016, p. 32 [Entrevista al autor por Ayșe Çavdar]. 
y la provisión de servicios en vez del reconocimiento de derechos, por otra.

Para explicitar tal planteamiento, en primer lugar, se realizará un breve recuento histórico de los flujos migratorios en Turquía. El propósito de esta primera parte es analizar cómo el Estado turco ha ido modificando su sistema migratorio para responder a una visión nacional, hacer frente a las características únicas de su posición geográfica y su pasado imperial e incorporar el derecho internacional, que, con reservas sustantivas, es parte fundamental del actual sistema de protección internacional. En segundo lugar, se explicarán las categorías del sistema de protección internacional actual y las dinámicas que intervinieron en su origen, legislación e implementación. En tercero, se analizará la estrategia general del gobierno para gestionar la crisis humanitaria como una emergencia de carácter temporal, lo que dio origen a una estructura institucional y administrativa abocada a la provisión de servicios, antes bien que al reconocimiento de derechos. Se establecerá, en fin, la relación entre el sistema de protección internacional y la estrategia de gestión de la crisis.

\section{DESARROLLO HISTÓRICO}

Se realizará, en este apartado, un análisis histórico para saber si ha habido una relación entre el sistema migratorio adoptado por Turquía, los flujos migratorios recibidos y la manera de gestionarlos. Asimismo, nos permitirá observar cómo el Estado turco concibió la migración en distintas épocas y las distintas maneras en las que clasificó a los migrantes según su estrategia política, para lo cual utilizaremos el esquema de Didem Daniş, quien identifica tres grandes periodos con que puede analizarse la migración en Turquía: ${ }^{9}$ el periodo nacio-

9 "A New Period in Turkish Migration Policy: Syrian Refugees as a Foreign Policy Instrument”, Saha. Citizen's Assembly-Turkey, núm. esp. 1, 2016, p. 27. 
nalista, que va desde la Tanzimat ('reorganización', en turco) hasta la Posguerra Fría; el periodo global, que se extiende hasta el 2011 con el inicio del influjo de sirios desplazados por la guerra y da origen al tercero de éstos, el periodo neotomano, que está en pleno desarrollo todavía.

\section{El periodo "nacionalista"}

El propósito fundamental, durante este periodo, era la turquificación de la población. Por los cambios históricos en él acaecidos, habrá que dividirlo en tres etapas: el Imperio Otomano (1839-1923), la Joven República (1923-1945) y la Guerra Fría (1945-1991).

Si bien durante la Tanzimat (1839) se abrió la migración a todos aquellos que rindieran alianza al Sultán y se mantuvo la libertad de religión, se favoreció y fomentó la inmigración de minorías turcas de regiones adyacentes (tártaros de Crimea, circasianos del Cáucaso, musulmanes de los Balcanes), ${ }^{10}$ proceso que se vio reforzado por la sostenida pérdida de territorio. Los recién llegados no fueron considerados migrantes, sino soydaşlar ('parientes') o muhajir (en alusión a quienes huyeron con Mahoma a Medina). ${ }^{11}$

Durante la Joven República, se reforzó la política nacionalista y se eliminó la relativa apertura previa con dos propósitos claros: asegurar las fronteras y profundizar la turquificación. Entre los hechos concretos de este período se pueden enumerar: la llegada de 384 mil turcos, la partida de 1.1 millones de griegos $^{12}$ (resultado del intercambio de población de 1922), y la llegada de más 800 mil muhajir de "raza y cultura turca" de los Balcanes entre 1923 y $1945 .{ }^{13}$ Se permitió, por lo demás, el ingreso de 100 mil judíos, que se refugiaron tempo-

${ }^{10}$ D. Latif, "Refugee Policy of the Turkish Republic", The Turkish Yearbook of International Relations, vol. 33, 2000, pp. 1-29.

${ }^{11}$ Daniş, art. cit., p. 27.

12 A. Mango, Atatürk, Nueva York, The Overlook Press, 2002, p. 390.

${ }^{13}$ Daniş, art. cit., p. 27. 
ralmente en Turquía, al huir de la Europa asolada por la Segunda Guerra Mundial. ${ }^{14}$

Durante la Guerra Fría, Turquía mantuvo la política nacionalista, aunque condicionada por su papel como aliado de Occidente cercano a los límites de la Unión Soviética. En esta etapa, la población turca se mantuvo homogénea, los flujos de refugiados que llegaban de la Europa Oriental no eran masivos, no tuvieron grandes dificultades para integrarse y Occidente estaba dispuesto a acogerlos. Esta situación no alteró el carácter de emisor de migrantes y continuó la migración de turcos hacia Europa por razones económicas. ${ }^{15}$ El hecho de que Turquía no se considerara un país de inmigración, según afirman İçduygu y Keyman, demoró el desarrollo de políticas migratorias efectivas. ${ }^{16}$

Se incorporó, en esta etapa, un primer cambio fundamental con la firma de la Convención de Ginebra de 1951 sobre el Estatuto de los Refugiados, la cual establecía dos limitaciones: una, geográfica (Europa) y otra, temporal (hasta el 1 de enero de 1951). En ocasión de su ratificación, Turquía no contempló la limitación temporal, aunque sí la geográfica. Si bien el Protocolo Relativo al Estatuto de los Refugiados de 1967 eliminó ambas restricciones, Turquía mantuvo, y mantiene hasta la fecha, la limitación geográfica. Según la ley turca, "alguien no europeo será considerado solicitante de asilo, pero no refugiado", ${ }^{17}$ lo que ha propiciado un sistema de doble nivel.

De un lado, un primer nivel para personas de origen europeo. Durante este período, el mayor flujo europeo vino de

${ }^{14}$ S. P. Elitok y T. Straubhaar, "Migration from Turkey to Europe: Betwixt and Between?" en Europe, Turkey and the Mediterranean Fostering Cooperation and Strengthening Relations. Europe in Dialogue, Berlín, Bertelsmann Stiftung, Gütersloh, 2012, pp. 97-106.

${ }^{15}$ A. İçduygu y E. F. Keyman, "Globalization, Security, and Migration: The Case of Turkey", Global Governance, vol. 6, núm. 3, 2000, p. 391.

${ }^{16}$ Ibidem., p. 384.

17 C. Soykan, "The New Draft Law on Foreigners and International Protection in Turkey" Oxford Monitor of Forced Migration, vol. 2, núm. 2, 2012, p. 41. 
Bulgaria en tres etapas: ${ }^{18} 156$ mil migrantes entre 1949 y 1951, 130 mil turcos búlgaros familiares de la primera oleada en los setenta y la última a fines de los ochenta. La tercera ola fue resultado de políticas persecutorias ${ }^{19}$ por las que 310 mil turcos búlgaros ingresaron al país. ${ }^{20} \mathrm{Al}$ principio, estuvieron en campos, pero tenían libertad para moverse, asentarse y trabajar. La mitad regresó a Bulgaria y muchos de los 240 mil que se quedaron se convirtieron en ciudadanos turcos. ${ }^{21}$ También ingresaron migrantes de los Balcanes (bosnios y kosovares que habían participado en guerrillas a favor del Eje durante la Segunda Guerra Mundial). De otro, un segundo nivel para personas de origen no europeo. Los mayores flujos en esta etapa provinieron de Irán e Iraq. Tras la revolución iraní, alrededor de 1.5 millones de iraníes ${ }^{22}$ entraron al país sin visa y pudieron quedarse temporalmente. La mayoría de ellos fueron reasentados en terceros países y sólo unos pocos obtuvieron la residencia.

Por lo que toca a los iraquíes, hubo dos grandes olas. La primera fue como consecuencia de la guerra entre Iraq e Irán. Luego de la matanza de Halabja, en 1988, los peshmergas kurdos ingresaron a Turquía y "algunos funcionarios creyeron que, bajo el derecho internacional, Turquía no tenía obligaciones para con estos refugiados. Prefirieron repatriarlos o reasentarlos en terceros países. ${ }^{23}$ La segunda crisis fue tras la Guerra del Golfo, cuando 467 mil iraquíes y personas de ter-

18 N. A. Ş. Önerand y D. Genç, "Continuity or Change in Turkey's Mass Migration Policy: From 1989 émigrés to Syrian «guests»", en G. Seker et al. (eds.), Turkish Migration Conference 2015, Selected Proceedings, Londres, Transnational Press London, p. 25.

${ }^{19}$ Loc. cit.

20 İçduygu y Keyman, art. cit., p. 390.

${ }^{21}$ K. Kirişci, "«To Lift or Not to Lift» the Geographical Limitation to the 1951 Geneva Convention Relating to the Status of Refugees: Turkey's Pre-Accession to the Eu and Asylum", borrador elaborado para la 4th METU International Relations, Ankara, 30 de junio-2 de Julio de 2005, en http:/ / edam.org.tr/document/Kirisci2.pdf, consultado el 2.XI.2017.

${ }^{22}$ Véase Latif, art. cit., p. 17.

${ }^{23}$ Ibid., p. 10. 
ceros países, que estaban en Iraq, trataron de cruzar la frontera. Turquía lo consideró una amenaza a su seguridad nacional, cerró sus fronteras y pidió la intervención del Consejo de Seguridad. Finalmente, los refugiados fueron establecidos en campos dispuestos en la frontera turco-iraquí.

Este sistema de doble nivel, fruto de la limitación geográfica, es fundamental para entender el sistema de protección internacional vigente aplicado a las personas procedentes de Siria. Tal limitación, por lo demás, es un argumento que utilizan no pocas ong para afirmar que Turquía no puede tenerse por un "país seguro" en el marco del acuerdo de readmisión firmado entre la Unión Europea (UE) y esos países.

\section{El periodo "global"}

Este período estuvo marcado por los grandes cambios de la Posguerra Fría y las consecuencias del proceso de globalización. El fenómeno migratorio en Turquía se volvió más complejo con la consolidación de tres flujos fundamentales, según afirman İçduygu y Keyman: ${ }^{24}$ 1) una mayor tasa de emigración a Europa; 2) el papel de Turquía como receptor de solicitantes de asilos; 3) la experiencia con la migración de tránsito. Además, el inicio del proceso de adhesión de Turquía a la UE, en 2005, dio lugar al planteamiento de una serie de reformas destinadas a adoptar el acervo comunitario, entre ellas la reforma del sistema migratorio y de protección internacional. En estas circunstancias, comienza a gestarse el proyecto de la ley de Extranjeros y Protección Internacional, que vio la luz en 2013.

En cuanto al origen de los flujos, según Elitok y Straubhaar, se estima que cerca de un millón de migrantes llegaron a Turquía tras la caída de la Unión Soviética. ${ }^{25}$ Según cifras de la Dirección General de Manejo de la Migración (DGMM), la

${ }^{24}$ İçduygu y Keyman, art. cit., p. 384.

${ }^{25}$ Elitok y Straubhaar, art. cit., p. 97. 
migración forzada provino mayoritariamente de los Balcanes, 20 mil refugiados provenientes de Bosnia (1992-1998), 17746 de Kosovo y 10500 de Macedonia (la mayoría ha regresado) ${ }^{26}$ $\mathrm{Al}$ mismo tiempo, Turquía comenzó a ser usada como vía de ingreso terrestre hacia la UE por migrantes de África y Asia, aunque por las dificultades muchos migrantes de tránsito se han quedado varados en ella. Otro gran cambio fue la política de mantener a las minorías turcas no europeas (azeríes, ahiska, chechenos y uzbekos) en sus lugares de origen como estrategia política, tanto por cuestiones de política doméstica como exterior.

\section{Periodo "neotomano"}

Éste, según Daniş, tuvo su origen en el flujo masivo de refugiados sirios y fue sustentado políticamente por "discursos conservadores otomanistas e islamistas". ${ }^{27} \mathrm{El}$ debate sobre la gestión de la crisis humanitaria y su fundamento discursivo se tratará líneas abajo antes del desarrollo del sistema de protección internacional vigente.

\section{SISTEMA LEGAL VIGENTE}

Si bien la crisis humanitaria aceleró el proceso final de elaboración de una ley sobre protección internacional para dar un marco normativo en medio de una crisis sin precedentes, la ley de Extranjeros y Protección Internacional (LEPI), promulgada de manera unánime por el Parlamento de Turquía en abril de 2013, fue el fruto de años de trabajo y colaboración entre Turquía, la ue, la sociedad civil turca y organismos internacionales (en especial, el ACNUR). Si bien la LEPI fue

26 DGmm, Türkiye ve GÖC, Ankara, Göç İdaresi Genel Müdürlüğü, 2015, p. 11.

${ }^{27}$ Art. cit., p. 27. 
parte del paquete de reformas para armonizar el marco legal turco con el acervo comunitario, ese proceso tuvo otros dos propósitos, que deben interpretarse nacionalmente: debía ser la pieza fundamental para estructurar los tres flujos anteriormente mencionados y, a la vez, dejar suficiente espacio de maniobra al gobierno para determinar el marco jurídico con el que se gestionaría el ingreso masivo de personas, sus condiciones de estancia y egreso del país.

La LEPI constituye el "marco legal para el asilo en Turquía y afirma las obligaciones de Turquía hacia las personas necesitadas de protección internacional, independientemente de su país de origen, a nivel de derecho interno" 28 y, más allá de las observaciones que se le puedan realizar a la luz de la situación actual y el sentido restrictivo que sostiene en términos de protección internacional, ha resultado ser un gran avance con respecto del marco jurídico anterior.

No se puede entender la LEPI y las regulaciones subsiguientes sin comprender, claro está, las circunstancias históricas. Luego del estallido de las protestas en contra del gobierno de Bashar al-Assad, en abril de 2011, un primer grupo de 252 sirios ingresó a Turquía por Hatay. En ese momento, el gobierno tomó dos decisiones que marcarían el camino de la política futura. Por un lado, definió el hecho como un "influjo masivo" (categoría inexistente en el sistema legal de entonces) ${ }^{29}$ y, por otro, abrió sus fronteras y aseguró que satisfaría las necesidades básicas de los "huéspedes" (término

28 Refugee Rights Turkey, "Asylum Information Database. Country Report: Turkey. European Council of Refugees and Exiles", 2015, p. 15, en http:/ / www.asylumineurope.org/reports/country/turkey, consultado el 10.II.2017.

${ }^{29}$ Según M. Corabatir (The Evolving Approach to Refugee Protection in Turkey: Assessing Practical and Political Needs, Washington, DC, Migration Policy Institute, 2016), el ACNUR se abstuvo de registrar peticiones individuales de los primeros sirios con el propósito de "apaciguar los temores del gobierno de que el acceso al reasentamiento del ACNUR socavara la política de campamentos al atraer a grandes multitudes esperanzadas con el reasentamiento al centro de procesamiento de Ankara" (p. 8). 
usado por Turquía para referirse a los refugiados sirios), no sin haber asegurado el principio de non-refoulement. Fue ésta la base del esquema inicial de protección temporal establecido en octubre de 2011. Desde entonces, el flujo ha sido continuo, con dos episodios de ingresos masivos, 190 mil kurdos sirios provenientes de Kobane en octubre de 2014 y unos 25 mil árabes, kurdos y turcomanos, que escaparon de la ofensiva de Tal Abyad, en junio de 2015. ${ }^{30}$

La falta de un marco jurídico integral al inicio de la crisis suscitó una estrategia fragmentada y carente de sistematicidad, que, de suyo, dio margen de acción a Ankara para desarrollar su estrategia política ante el gobierno sirio y para hacer frente a la crisis. El sistema vigente anterior a la LEPI, es decir la Regulación del Asilo de 1994, establecía que los flujos masivos debían ser "frenados en la frontera", a menos que hubiese una "instrucción gubernamental en contra". ${ }^{31}$ Aunque esta orden se dio, esa protección temporal dependía, desde su concepción, de cierta discrecionalidad política y administrativa.

\section{Categorías de protección internacional}

Analizar las categorías de protección internacional del sistema actual ayudará a comprender mejor la relación entre dicho sistema y la estrategia de Turquía para gestionar esta crisis -y cualquiera que pueda surgir en el futuro. La LEPI logró un equilibrio delicado entre los principios del acervo comunitario exigidos por la ue y la flexibilidad que Turquía consideraba necesaria para afrontar crisis políticas y humanitarias, ejemplo de lo cual es la vigencia de la limitación geográfica. La LEPI establece tres tipos de protección internacional individual. Por la "limitación geográfica", según se apuntó líneas arriba, se mantiene el modelo de doble nivel.

${ }^{30}$ Refugee Rights Turkey, “Asylum Information Database...”, p. 109.

${ }^{31}$ Ibid., p. 104. 
1) Europeos. Éstos pueden calificarse como refugiados, según los términos del Artículo 1 de la Convención de Ginebra de $1951 .^{32}$

2) No europeos. Los solicitantes de asilo ${ }^{33}$ no europeos, como éstos, pueden solicitar protección internacional de forma individual y pueden ingresar en una de estas dos categorías:

a) Refugiado condicional. Coincide con la definición de refugiado, pero no puede adquirir ese estatuto a causa de la limitación geográfica. Tiene menos derechos que un refugiado; no tiene derecho a la integración en el largo plazo ni derecho a la reunificación familiar. Como explican Sari y Dinçer, esta categoría requiere un trámite doble, pues la DGMM sólo concede estatuto de refugiado condicional y el ACNUR, el estatuto de refugiado, según la Convención y, por tanto, el derecho al reasentamiento en un tercer país. ${ }^{34}$ El AGNUR mantie-

32 Es refugiado todo aquel que "debido a fundados temores de ser perseguido por motivos de raza, religión, nacionalidad, pertenencia a determinado grupo social u opiniones políticas, se encuentre fuera del país de su nacionalidad y no pueda o, a causa de dichos temores, no quiera acogerse a la protección de tal país; o que, careciendo de nacionalidad y hallándose, a consecuencia de tales acontecimientos, fuera del país donde antes tuviera su residencia habitual, no pueda o, a causa de dichos temores, no quiera regresar a él" (Art. 1 a/2).

${ }^{33}$ Según el ACNur, un solicitante de asilo es una persona que pide que se la reconozca como refugiada, pero cuyo reconocimiento como tal aún no es definitivo hasta tanto termine el proceso de Determinación del Estatuto de Refugiado (DER).

34 "Toward a New Asylum Regime in Turkey?", Movements: Journal of Critical Migration and Border Regimes Studies, vol. 3, núm. 2, 2017, pp. 66-67. Una vez obtenido el estatuto de refugiado emitido por ACNUR, comienza el proceso de restablecimiento, que puede llevar años (hasta cuatro) y que, como tal, no es un derecho y puede ser denegado por un potencial país receptor (que aplica sus propios criterios de aceptación). Por último, tras ser admitidos para restablecimiento, deben recibir el permiso de salida de las autoridades turcas (lo que implica la obligación de haberse registrado y haber sido reconocido como refugiado condicional por la DGMM). Véase p. 67 del artículo de Sari y Dinçer. 
ne un papel clave como actor complementario y sigue realizando la Determinación del Estatuto de Refugiado (DER). Sin embargo, las decisiones del ACNUR no tienen efectos vinculantes bajo la LEPI.

b) Protección subsidiaria. Se refiere a "personas que de otro modo podrían estar sujetas a pena de muerte o tortura en su país de origen o correr un «riesgo personalizado de violencia indiscriminada» debido a situaciones de guerra o conflicto armado interno". 35 Tienen estas personas menos derechos que un refugiado, pues no pueden establecerse en el país, pero tienen derecho a reunificación familiar. Esta protección existe para las personas que deben huir como consecuencia de una violencia generalizada o del colapso del orden público, pero que no califican como refugiado. ${ }^{36}$ Protegerlas es un deber del Estado según el Derecho Internacional de los Derechos Humanos y el Derecho de Guerra.

La ley prevé complementariamente el concepto de protección temporal en casos de "influjo masivo" de refugiados (Artículo 91 ${ }^{37}$ ). Kemal Kirişci apunta que se trata de asilo masivo, cuando

cantidades muy grandes de refugiados cruzan las fronteras como resultado de violaciones masivas a los derechos humanos y violencia sistemática, mientras que el caso de las solicitudes individuales de asilo, se refieren a situaciones en las que las personas que enfrentan una persecución huyen de sus países de origen y buscan refugio en otro país. Los derechos y obligaciones de los solicitantes individuales de asilo y refugiados es-

${ }^{35}$ Refugee Rights Turkey, “Asylum Information Database...”, p. 17.

${ }^{36}$ Durukan, entrevista cit., p. 31.

${ }^{37}$ Artículo 91 (1) de la LEPI: "Se podrá proporcionar protección temporal a los extranjeros que, habiendo sido forzados a dejar su país y no pudiendo regresar al país que han dejado, hayan llegado en masa a las fronteras de Turquía o las hayan cruzado buscando protección temporal y de emergencia". 
tán reguladas por la Convención de Ginebra de 1951, mientras que los casos de asilo en masa están definidos por decisiones del Comité Ejecutivo del ACNUR y el derecho internacional humanitario general. ${ }^{38}$

El concepto proviene de la legislación europea y es una solución provisional, según la cual, si se da un ingreso masivo, no es necesario realizar una evaluación individual. ${ }^{39}$ Sin embargo, la ley no establece procedimientos, alcances y contenido, sino que delega la elaboración de una regulación al Consejo de Ministros (Artículo 91, 2, de la LEPI). Aquí radica la clave de la LEPI y su relación con la estrategia de manejo de crisis del gobierno turco. La ley plantea una primera limitación en cuanto a la certidumbre y alcance de los efectos de la protección temporal a partir de la capacidad legislativa de que se dota al Consejo de Ministros. El proceso institucional requiere menos negociación, el diálogo es más acotado y los derechos son más endebles, dado que la regulación puede modificarse sin contrapeso parlamentario.

$\mathrm{Al}$ margen de las categorías plasmadas en la ley, hay dos regímenes especiales. Uno, de protección temporal de carácter general aplicado actualmente a los refugiados sirios y otro, para los iraquíes. De más no está recordar aquí que por cuanto atañe a Turquía en ninguno de los dos casos se trata de refugiados.

La Regulación de la Protección Temporal (RPT) entró en vigor en octubre de 2014 y es actualmente el instrumento legal fundamental del sistema de protección temporal que se aplica a los sirios. Al ser una resolución ministerial, "la protección temporal dentro del marco de la RPT no se define como una forma de protección internacional, sino como una medida complementaria usada en situaciones donde el

${ }^{38}$ K. Kirişci, Syrian Refugees and Turkey's Challenges: Going Beyond Hospitality. Washington, D.C.: The Brookings Institution, 2014, p. 8, en https:// www.brookings.edu/wp-content/uploads/2016/06/Syrian-Refugeesand-Turkeys-Challenges-May-14-2014.pdf, consultado el 2.XI.2016.

${ }^{39}$ Durukan, entrevista cit., p. 32. 
procesamiento de la elegibilidad para protección internacional individual es considerado impráctico". ${ }^{40}$

Desde el punto de vista legal, los sirios que están en Turquía deberían ser incluidos naturalmente en la categoría de protección subsidiaria de la LEPI, toda vez que han huido de un "estado de violencia generalizada" y porque quienes son perseguidos de manera individual podrían acceder a la categoría de refugiado condicional, aunque sin la condición de refugiado. ${ }^{41}$ Sin embargo, Turquía ha creado un estado excepcional para los sirios, a quienes se permite permanecer legalmente en el país y tener acceso a servicios de salud, educación y trabajo -no sin límites-, la garantía de non-refoulement ${ }^{42}$ y la reunificación familiar (aunque el fraseo de la regulación no indica estrictamente tal derecho, el cual está sujeto a la discreción de la DGMM). ${ }^{43}$

Los beneficiarios de la regulación son los refugiados provenientes de Siria, considerados como una población que ingresó masivamente, y palestinos apátridas provenientes de Siria, que llegaron a Turquía a partir del 28 de abril de 2011. Sin embargo, hay excepciones: los sirios que arribaron antes del 28 de abril y ya solicitaron asilo; los sirios que no llegaron directamente de Siria; ${ }^{44}$ los sirios que ingresan por segunda vez y quieren ser acogidos una vez más por la protección temporal; ${ }^{45}$

${ }^{40}$ Refugee Rights Turkey, “Asylum Information Database...”, p. 104.

${ }^{41}$ Durukan, entrevista cit., p. 31.

42 Refugee Rights Turkey, “Asylum Information Database...”, p. 15.

43 Ibid., p. 136.

${ }^{44}$ Las frases de la regulación "que llegaron a nuestras fronteras" o "han cruzado nuestras fronteras" se interpretan a partir de la connotación de 'frontera terrestre': las personas deben llegar directamente de Siria y no a través de un tercer país. De otro modo, si tienen pasaporte válido pueden solicitar residencia temporal como cualquier extranjero o acogerse a la protección temporal (como otros solicitantes de asilo). Empero, la DGMM podría no admitirlos por provenir de un "tercer país seguro" o con base en el concepto de "primer país de asilo". Cf. Refugee Rights Turkey, “Asylum Information Database...”, p. 111.

45 Esto aplicaría, por ejemplo, a los sirios que tratan de salir legal o ilegalmente de Turquía y luego vuelven a ingresar. 
los sirios con permiso de residencia legal que se ven forzados a refugiarse en la protección temporal, no bien se vence su pasaporte y no pueden renovar su residencia; y los nacionales de terceros países, en fin, que llegan de Siria.

En el caso de los iraquíes (el grupo más grande de solicitantes individuales de asilo desde 2007), la crisis comenzó con la llegada de entre 40 y 50 mil yazidíes que huyeron de Mosul en junio de 2014. Para enero, eran entre 200 y 250 mil, muchos de los cuales no estaban registrados en la DGMM, ni ante el ACNUR. Tras varias medidas, la DGMM implementó una ruta $a d$ hoc por la que los iraquíes que buscaran protección internacional urgente, ya fuera de manera individual o masiva, ya no serían procesados como protección internacional. ${ }^{46}$ En febrero de 2015 , ya había iraquíes registrados en la DGMM con un "permiso de residencia humanitaria" fuera del sistema de protección internacional. Actualmente tienen la opción de solicitar protección internacional o de obtener el "permiso de residencia por razones humanitarias", lo que implica un trato como el otorgado a otros extranjeros residentes en el país.

\section{CONSECUENCIAS JURÍDICAS Y ADMINISTRATIVAS DEL SISTEMA DE PROTECGIÓN TEMPORAL}

La complejidad del tema hace imposible desligar las consecuencias legales analizadas de las políticas, sociales, económicas y culturales del sistema de protección internacional vigente. Sin embargo, por los límites planteados en las primeras líneas de este trabajo, nos abocaremos a estudiar las implicancias políticas y jurídicas del sistema, para luego establecer la relación con la estrategia de gestión de la crisis del gobierno turco.

${ }^{46}$ Refugee Rights Turkey, “Asylum Information Database...”, 69. 
Implicancias jurídicas: concesiones, no derechos

Hay dos garantías básicas en el caso de refugiados o personas que huyen de un país sumido en la violencia sistemática: la garantía de admisión y el non-refoulement. ${ }^{47}$ En el caso de la garantía de admisión, hay dos elementos por analizar en el presente marco jurídico: la gobernación provincial puede impedir el acceso a alguien sin pasaporte válido y Turquía se reserva la posibilidad de "tomar medidas adicionales concernientes al movimiento masivo de personas a lo largo de la línea fronteriza de Turquía o más allá de la línea fronteriza de Turquía”, ${ }^{48}$ lo cual limita las garantías básicas, toda vez que el gobierno puede cerrar las fronteras cuando lo desee. De hecho, desde 2014, la actitud de Turquía para con los refugiados provenientes de Siria comenzó a cambiar, porque privilegió las cuestiones de seguridad sobre las humanitarias, la política de "puertas abiertas" terminó definitivamente y se comenzó progresivamente a blindar la frontera. ${ }^{49}$ En 2016, se implementó una visa para sirios que ingresaban por aire o mar $^{50}$ y el gobierno dejó de registrar nuevos arribos -salvo casos de emergencia-, dejando a los nuevos refugiados sin la capacidad de reclamar los servicios provistos bajo la protección temporal de los sirios que habían llegado durante los años anteriores. ${ }^{51}$ Previstas estas situaciones, Refugee Rights

${ }^{47}$ M. İneli Ciğger, "Implications of the New Turkish Law on Foreigners and International Protection and Regulation no. 29153 on Temporary Protection for Syrians Seeking Protection in Turkey", Oxford Monitor of Forced Migration, vol. 4, núm. 2., 2014, p. 51, en http://oxmofm.com/vol4-no-2/, consultado el 4.II.2017.

${ }^{48}$ Refugee Rights Turkey, “Asylum Information Database...”, p. 109.

${ }^{49}$ G. Heck y S. Hess, "Tracing the Effects of the EU-Turkey Deal: The Momentum of the Multi-layered Turkish Border Regime", Movements: Journal of Critical Migration and Border Regimes Studies, Múnich, vol. 3, núm. 2, 2017, p. 44.

${ }^{50}$ Loc. cit.

51 F. Baban et al., "Playing Border Politics with Urban Syrian Refugees: Legal Ambiguities, Insecurities, and Humanitarian Assistance in Turkey", Movements: Journal of Critical Migration and Border Regimes Studies, Múnich, vol. 3, núm. 2, 2017, p. 92. 
Turkey afirma que el principio de non-refoulement debe ser el paraguas conceptual para interpretar esas provisiones.

Otro punto por analizar en el caso de la garantía de admisión es que las personas que no llegan por tierra desde Siria, como se mencionó líneas arriba, podrían sufrir el rechazo de su solicitud de asilo a partir de los principios de "primer país de asilo" o "tercer país seguro" ${ }^{52}$ Esto dos conceptos están sujetos tanto a una interpretación jurídica dinámica, como a criterios establecidos por los países al momento de evaluar la admisibilidad de las solicitudes de asilo. El concepto primer país de asilo se refiere a un país donde el solicitante ya fue previamente reconocido como refugiado, por lo que aún puede invocar esa protección o puede "disfrutar una protección suficiente y efectiva incluyendo la protección contra el refoulement". ${ }^{53}$ Un tercer país seguro es un país distinto del país de origen del solicitante de asilo y del país en el que se encuentra, donde la persona no corre peligro de persecución por su raza, religión, nacionalidad o pertenencia a un grupo social u opinión política, tiene la garantía de non-refoulement y puede solicitar el estatuto de refugiado según los términos de la Convención de 1951, sin correr el riesgo de ser sometido a un daño grave. ${ }^{54}$

Dadas las circunstancias legales, políticas y administrativas de Turquía, es necesario indagar cómo la regulación actual afecta las garantías contra la detención y la expulsión (non-refoulement). Según Soykan, los extranjeros no pueden ser detenidos por solicitar asilo, aunque sí por violar la regulación vigente (por ejemplo, por no presentarse al control de residencia en la ciudad donde deben residir). ${ }^{55}$ Otro problema, que Soykan señala, no menos que algunas ONG, como Mülteci-Der, es que a muchos extranjeros detenidos por las fuerzas de seguridad, luego de haber infringido la ley migra-

${ }^{52}$ Refugee Rights Turkey, “Asylum Information Database...”, p. 67.

53 Ibid., p. 64.

${ }^{54}$ Ibid., p. 65.

${ }^{55}$ Art. cit., p. 43. 
toria, no se ofrece solicitar asilo y se los retiene hasta por un año en centros de deportación. ${ }^{56}$

En cuanto a la libertad para salir de Turquía, los sirios acogidos bajo el sistema de protección temporal deben pedir autorización de la DGMM, que emite un permiso de salida, ya por visita temporal, ya por manera permanente. Cabe agregar que, desde enero de 2017, si un sirio bajo protección temporal viaja de Turquía a otro país, su protección será cancelada, ${ }^{57}$ lo cual hace que las posibilidades de dejar el país estén a merced de la autoridad administrativa, por lo que su libertad de movimiento está limitada. Por lo demás, tampoco pueden solicitar ni protección internacional, ni el consiguiente reasentamiento con el AGNur. ${ }^{58}$ En el caso de los sirios que no están bajo protección temporal, pueden salir del país libremente, pero no son elegibles para reasentamiento por el ACNUR.

El tercer tema fundamental, la posibilidad de integración futura de los sirios bajo protección temporal, está en el centro de la relación entre el sistema de protección internacional o la noción de temporalidad en la gestión de la crisis. Actualmente, la regulación no admite solicitar asilo de manera individual una vez terminada la protección temporal, excluyendo a los beneficiarios de una potencial integración definitiva en Turquía. El documento de identificación de los sirios (kimlik) no es un permiso de residencia y el tiempo vivido en Turquía bajo protección temporal no cuenta como requerimiento de residencia continua de cinco años para solicitar la ciudadanía turca.

56 Véase Mülteci-Der, Mülteci-Der's Observations on Refugee Situation in Turkey, Izmir, 13 de mayo de 2016, en http://www.multeci.org.tr/en/haberler/multeci-ders-observations-on-refugee-situation-in-turkey, consultado el 12.V.2017.

${ }^{57}$ Baban et al., p. 84.

58 Los sirios pueden acceder al programa de reasentamiento del ACNUR por indicación de la DGMM, si se los considera personas vulnerables (mujeres, niños, LGBT, minorías étnicas, etc.). 
Implicancias administrativas: discrecionalidad

Además de las implicancias jurídicas, la ley propicia una vulnerabilidad adicional a partir de los amplios poderes que ésta, junto a la mencionada RPT, otorga al Consejo de Ministros, que puede:

1) Determinar quiénes son los beneficiarios.

2) Limitar o no la protección temporal a ciertas partes del territorio.

3) Decidir limitaciones a las medidas de protección temporal vigentes, suspenderlas temporal o definitivamente en caso de amenaza a la seguridad nacional, al orden público, a la seguridad pública o a la salud pública. Por ejemplo, tras quejas de la población local, algunos refugiados sirios fueron trasladados a otras ciudades o llevados a campamentos. ${ }^{59}$

4) Determinar el trato a los beneficiarios de la protección temporal y las medidas con respecto de ellos. No garantiza un explícito e irrestricto derecho al trabajo, a la educación y a la asistencia social.

5) Decidir el comienzo, duración y fin de la protección. Según İneli Ciğer, una protección temporal sin límite de tiempo podría socavar la Convención de 1951, al "sugerir una alternativa a ella para la protección de refugiados, en lugar de ser sólo una respuesta de emergencia en situaciones de influjo masivo". ${ }^{60}$ La protección temporal normalmente termina, cuando se alcanza un tiempo límite o se dan ciertos requisitos que permiten la repatriación o la integración de los refugiados en la sociedad que los alberga. En el primer caso, según el artículo 42, las autoridades turcas podrían

${ }^{59}$ U. Korkut, "Pragmatism, Moral Responsibility or Policy Change: The Syrian Refugee Crisis and Selective Humanitarianism in the Turkish Refugee Regime", Comparative Migration Studies, vol. 4, núm. 2, 2016, p. 2, en https://comparativemigrationstudies.springeropen.com/articles/10. 1186/s40878-015-0020-9, consultado el 12.V.2017.

${ }^{60}$ Art. cit., p. 31. 
facilitar y apoyar las repatriaciones voluntarias. Pero, en caso de terminar la protección temporal, el Consejo podría ordenar el regreso de los sirios a su país de origen (podría llegar a implicar una violación del non-refoulement), conceder a los grupos una protección grupal, solicitar asilo individual o permanecer en Turquía bajo los términos de la LEPI. Potencialmente, "el Consejo podría ordenar el regreso de todos los sirios sin considerar las cláusulas de cesación provistas en la Convención de Refugiados de 1951. ${ }^{61}$

6) Reglamentar la libertad de movimiento. A la inversa de los refugiados condicionales, que están obligados a residir en ciudades satelitales, ${ }^{62}$ los sirios, en principio, podían moverse libremente por el país. Sin embargo, a partir de agosto de 2015 y haciendo uso de la discrecionalidad que le confiere la LEPI, "el Ministerio del Interior ordenó a las gubernaturas controlar el movimiento de los sirios, posiblemente para frenar su movimiento hacia el oeste rumbo a la UE". ${ }^{63}$

En conclusión, independientemente de la concepción de la ley y la regulación, el elemento fundamental es el espacio que tiene el gobierno para determinar elementos claves del sistema de protección temporal, así como su articulación, según sus objetivos políticos ante fenómenos coyunturales exógenos y endógenos. Según se ha mencionado, el sistema de protección temporal puede ser modificado sustancialmente

${ }^{61}$ Ibid., p. 33.

${ }^{62}$ Véase K. S. Biehl, "Governing through Uncertainty: Experiences of Being a Refugee in Turkey as a Country for Temporary Asylum", Social Analysis, vol. 59, núm. 1, 2015, pp. 57-75. La DGMM es la encargada de hacer una lista de "ciudades satélites" a partir de la evaluación de la distribución de población y de otras consideraciones. Luego comunica al ACNUR las provincias a las que deben ser referidos los nuevos solicitantes. Actualmente, hay 50 ciudades que están en el interior del país, lejos de la costa del Mediterráneo y de las fronteras con Europa, para controlar y manejar a la población de solicitantes de asilo $(i d$. $)$. Las grandes zonas metropolitanas como Estambul, Ankara y Esmirna no son ciudades satélites.

${ }^{63}$ Çorabatir, op. cit., p. 12. 
por una decisión del Consejo de Ministros, lo cual plantea un cambio fundamental, pues, como afirma Ayşe Çavdar, "es una política con final abierto... Como tal es convertir el estado de excepción en regla”. 64

\section{ESTRATEGIA GUBERNAMENTAL}

ANTE LA CRISIS HUMANITARIA

El sistema de protección temporal derivado de la LEPI fue desarrollado e implementado como parte fundamental de una estrategia de manejo de crisis. Para escrutar la relación entre el sistema legal y dicha estrategia, se analizará la estructura institucional de gestión de la crisis, así como el discurso gubernamental que ha enmarcado la política de refugiados, en particular dos principios fundamentales emanados de ella: asistencia y temporalidad.

El núcleo de la estructura institucional para gestionar la crisis de los refugiados se ha mantenido estable a lo largo de la misma, a pesar del cambio de una política de alojamiento en campamentos fronterizos a una política de libre asentamiento. En este punto, Turquía ha emprendido una estrategia única al instalar y manejar sus propios campamentos, favoreciendo a su vez el autoasentamiento y diferenciándose del Líbano, el cual ha permitido que la ayuda humanitaria internacional instalara sus propios campamentos, y de Jordania, que ha optado por establecer un gran campamento operado por Naciones Unidas. ${ }^{65}$ Cabe decir que esta política fue alabada por el Banco Mundial, cuando afirmó que "integrar el apoyo a los refugiados en la corriente principal de provisión de servicios del gobierno puede ser más efectiva en tér-

64 Durukan, entrevista cit., p. 35.

${ }^{65}$ Refugee Studies Center, "The Syrian Humanitarian Disaster. Disparities in Perceptions, Aspirations and Behaviour in Lebanon, Jordan and Turkey", Rsc Research in Brief 3, University of Oxford, diciembre de 2015, p. 2. 
minos de costos que instalar campamentos a gran escala y canales de servicios de ayuda humanitaria paralelos". 66

La institución central de la estrategia gubernamental ante la crisis es la Agencia de Manejo de Emergencias y Desastres de Turquía (AFAD, según sus siglas en turco), que opera bajo la Oficina del Primer Ministro. Como menciona Korkut, "la participación activa (de la AFAD) ilustró el intento del gobierno de centralizar la crisis de refugiados bajo la égida del entonces Primer Ministro Erdoğan", ${ }^{67}$ pero también es un indicativo de que la estrategia buscó enmarcar la crisis como una situación temporal, ante la cual se debían proveer ciertos servicios de emergencia y no como un conflicto prolongado que podría hacer necesaria la implementación de políticas de integración definitiva. Lo resume Korkut de este modo: "el gobierno turco maneja las operaciones de asistencia a los refugiados con una institución que se ocupa de los desastres. Los desastres son, en esencia, temporales". 68

Desde el inicio de la crisis, la AFAD asumió la responsabilidad de instalar y administrar los campamentos, no sin la ayuda de la Media Luna Roja, y de todo lo relacionado con la provisión de servicios de los refugiados, incluidos su registro $^{69}$ y la emisión de documentos de identificación, hasta que esta función pasó a la DGMM con la promulgación de la LEPI. Aún está a cargo de los gastos de las operaciones de ayuda, incluidas las transferencias a gobiernos locales. A pesar de la centralidad de la AFAD, conforme fue avanzando la crisis y el gobierno pasó a una estrategia de autoasentamiento, el sistema se volvió más complejo y ahora incluye los mi-

${ }^{66}$ The World Bank, "Turkey's Response to the Syrian Refugee Crisis and the Road Ahead", p. 5, en www.worldbank.org.

${ }^{67}$ Art. cit., p. 9.

${ }^{68}$ Loc. cit.

${ }^{69}$ Como parte de la estrategia del gobierno para mantener el control de la crisis y del manejo de los refugiados, ni ACNUR ni ningún otro organismo internacional de ayuda humanitaria tuvieron participación en el registro de los refugiados que luego entrarían bajo el sistema de "protección temporal". 
nisterios de Salud, Interior, Defensa, el Consejo Superior de Educación y la Dirección de Asuntos Religiosos, así como gobiernos provinciales y locales.

Desde el punto de vista de la protección temporal, la creación de la DGMM como un organismo principal en materia de migración y asilo dependiente del Ministerio del Interior se consideró un gran avance. La autoridad recaía antes sobre el Departamento de Extranjeros de la Policía Nacional. El Consejo de Ministros con su facultad reglamentaria de la RPT, y la DGMM (Ministerio del Interior) en su capacidad de organismo central, asientan un gran poder de gestión de crisis e implementación de medidas fuera del alcance y el control del Parlamento, todo lo cual fue reforzado por la actual vigencia del Estado de Emergencia.

Esta dimensión institucional se vio apoyada desde los inicios de la crisis por un claro discurso gubernamental con alcances políticos internos (el fortalecimiento de la base conservadora religiosa) y regionales (la proyección de Turquía como actor regional hegemónico, lo que Daniş llama neotomanismo). Si bien estas afirmaciones merecen un análisis que excede el propósito de estas líneas, la gestión de la crisis se asienta en un discurso gubernamental que exalta la generosidad del pueblo turco y encumbra su papel como el protector de sus hermanos perseguidos, el centro está en la responsabilidad histórica y moral, más que en el humanitarismo. ${ }^{70}$ De hecho, se ha promovido activamente que el papel de los turcos para con los sirios sea igual al de los ensar, los musulmanes de Medina que apoyaron a los musulmanes que huyeron de La Meca, situación que, por cierto, también fue temporal. ${ }^{71}$

Más allá de las consecuencias e instrumentalizaciones de este discurso, hay dos elementos en él que marcan una complementariedad entre el marco legal de la protección temporal y la estrategia basada en la asistencia más que en el reconoci-

${ }^{70}$ Korkut, art. cit., p. 13.

${ }^{71} \mathrm{Id}$. 
miento de derechos, y en la temporalidad más que en la posible integración de los refugiados sirios.

El enfoque de asistencia soslaya la idea del derecho de las personas de buscar refugio y lo convierte en una concesión generosa del país de acogida, sujeta a la voluntad del último. Şenay Özden plantea que "nadie mencionó que buscar refugio es un derecho humano básico y actuó sobre este derecho. La perspectiva de la ayuda tampoco está basada en derechos humanos. Esto crea una jerarquía entre ciudadanos por un lado y refugiados por el otro". ${ }^{72}$ Tras esta estela, Korkut sostiene que

la protección temporal para los sirios opera esencialmente no basada en derechos formales, sino más bien en el discurso de generosidad del público [sic] turco. Al mismo tiempo, le indica al público $[s i c]$ turco que los sirios son una presencia temporal en Turquía. No obstante, ese enfoque caritativo en vez de uno basado en derechos formales alimenta la opinión negativa del público $[s i c]$ en Turquía hacia los refugiados. ${ }^{73}$

Desde el punto de vista jurídico, según Murat Erdoğan, las regulaciones existentes "no están basadas en los «derechos» de los refugiados y la «obligación» impuesta a los Estados en este sentido, sino más bien en «el apoyo bien intencionado del anfitrión ante los huéspedes»-dentro de los límites de las posibilidades". ${ }^{74}$ De ello es prueba que los campamentos fronterizos de Turquía son llamados oficialmente "campamentos de huéspedes", antes bien que "campamentos de refugiados". ${ }^{75}$

72 "The Opposition Failed Bitterly in the Refugee Issue", Saha. Citizen's Assembly Turkey, núm. esp. 1, 2016, p. 38 [Entrevista al autor por Ayşe Çavdar].

${ }^{73}$ Art. cit., p. 6.

${ }^{74}$ Urban Refugees from "Detachment" to "Harmonization". Siryan Refugees and Process Management of Municipalities: The Case of Istanbul, Estambul, Marmara Belediyeler Birliği, 2017, p. 18.

${ }^{75}$ Özden, "Syrian Refugees in Turkey", MPC Research Report 2013/05, Badia Fiesolana, Migration Policy Center (MPC)-European University Institute-Robert Schuman Center for Advanced Studies, 2013, p. 5. 
Por tanto, si se parte de la idea de un sistema de protección temporal prolongado sine die y una estrategia basada en la ayuda humanitaria y no en el derecho de solicitar refugio, se abre la puerta a una política centrada exclusivamente en la provisión de servicios. A propósito, Ayselin Yıldız y Elif Uzgören afirman que

la regulación de 2014 aborda el acceso a la salud, a la educación, al empleo y a otros tipos de asistencia social como "servicios" y le da al Estado el mandato de decidir cuáles deberían ser otorgados como "derechos" a las personas que necesitan protección... Este dilema de mantener la limitación geográfica, por una parte, y albergar de facto a tantos refugiados, por otra, deja a los refugiados en Turquía a merced de las autoridades turcas en términos de acceder a sus derechos fundamentales en línea con los principios universales. ${ }^{76}$

La temporalidad ha sido el otro eje de la estrategia que suscita varios dilemas al momento de aplicar medidas relacionadas especialmente con la educación y el acceso al mercado laboral. El concepto de huésped, aplicado a los refugiados sirios, implica que están un tiempo y luego se van a ir, por lo que no es necesario apurar la integración (en el sistema jurídico en particular y en las políticas públicas en general). A pesar de lo dicho, entrados como estamos en el séptimo año de la crisis y con pocas perspectivas de una pronta solución, comienza a percibirse malestar en ciertos sectores sociales y cambios en pronunciamientos políticos ante una realidad que marca dos vías claras: la repatriación (prematura o eventual) o el posible viraje hacia una política de integración y permanencia de parte de la población siria bajo protección temporal. De ello fue ejemplo la controversia desatada ante

76 "Limits to Temporary Protection: Non-Camp Syrian Refugees in İzmir, Turkey”, Southeast European and Black Sea Studies, vol. 16, núm. 2, 2006, p. 200, en http://www.tandfonline.com/doi/abs/10.1080/146838 57.2016.1165492?journalCode=fbss20, consultado el 8.II.2017. 
la idea del potencial otorgamiento de ciudadanía a ciertos nacionales de Siria y la integración de los niños sirios al sistema educativo formal turco.

Un estudio cuantitativo realizado en 2014 por Murat Erdoğan para la Universidad de Hacettepe sobre la percepción de la sociedad ante los refugiados sirios arrojó datos interesantes sobre las perspectivas de integración: el $76.5 \%$ de las personas encuestadas pensaban que "la presencia de sirios en Turquía podría causar problemas" 77 y ante la pregunta sobre la concesión de ciudadanía a sirios sólo el $10.8 \%$ se mostró de acuerdo (con una desaprobación del $81.7 \%) .{ }^{78}$

Independientemente de la estrategia basada en los pilares de asistencia y temporalidad, la crisis de los refugiados sirios parece seguir, en opinión de Ahmet İçduygu y Evin Millet, ${ }^{79}$ las etapas de otros movimientos migratorios: admisión, asentamiento, integración y naturalización. Por tanto, continúan los autores, "actualmente, el manejo de los refugiados sirios no es una cuestión de impedir la huida de refugiados y revertir su movimiento, sino que requiere acciones prácticas para proveerles mejores oportunidades de integración y asentamiento". ${ }^{80}$ Por otra parte, hay que considerar que el estado de temporalidad permanente aumenta la vulnerabilidad de los refugiados en general y de los sirios en particular, que "quedan con la sombría elección entre condiciones difíciles como refugiados urbanos, la libertad restrin-

77 Syrians in Turkey: Social Acceptance and Integration Research, Hacettepe University Migration and Politics Research Centre (HUGO), noviembre de 2014, p. 35, en https:/ / mmuraterdogan.files.wordpress.com/2014/12/ hugo-report-syrians-in-turkey-social-acceptance-and-integration-november-2014-04122014-en1.pdf, consultado el 27.XII.2017.

78 Ibid., p. 37.

79 "Syrian Refugees in Turkey: Insecure Lives in an Environment of Pseudo-Integration” (Working paper, 13), Roma, Istituto Affari Internazionali, Istanbul Policy Center y Mercator Foundation, 2016.

${ }^{80}$ Ibid., p. 3. 
gida de los campamentos de refugiados o continuar el viaje irregular hacia la UE". ${ }^{81}$

\section{Consideraciones FinAleS}

Luego de haber analizado el sistema de protección internacional de Turquía, podemos concluir que, si bien en un gran avance con respecto de la regulación anterior, el país todavía tiene un largo camino por recorrer para proveer un marco jurídico igualitario (por medio de la eliminación de la limitación geográfica), basado en derechos (no en la asistencia o la generosidad política) y que permita la integración de los acogidos bajo protección internacional (trascendencia del concepto de huésped temporal).

Se ha comprobado que este sistema de protección internacional -en especial, la protección temporal-complementa la estrategia gubernamental de concebir la crisis siria como una crisis temporal que debe ser tratada como una emergencia, descartando, en principio, políticas de largo plazo que impliquen la integración de los refugiados en la sociedad turca. Esta estrategia hace que el sistema de protección internacional tenga como característica fundamental lo que Biehl denomina "incertidumbre prolongada" a causa de "la espera indefinida, el conocimiento imperfecto y la volatilidad del estatus legal". ${ }^{82}$

Todos estos desafíos no deben hacernos perder de vista el enorme esfuerzo que está realizando el pueblo turco para asistir a millones de personas que huyen de una muerte segura ante la actitud pasiva de la comunidad internacional. Debemos tener en cuenta que Turquía tiene numerosos desafíos internos y los recursos para afrontarlos no son ilimitados, sobre todo a la luz de las circunstancias políticas internas y de

${ }^{81}$ C.orabatir, op. cit., p. 1.

${ }^{82}$ Biehl, art. cit., p. 58. 
las tensiones religiosas, étnicas e ideológicas que este conflicto está exacerbando entre su población.

Otro tema fundamental es la demanda que suele hacerse al Estado turco para que levante la limitación geográfica. Según explican Ayselin Yıldız y Elif Uzgören, "el caso sirio demostró que la reticencia de Turquía a levantar la limitación geográfica es entendible hasta cierto punto, como respuesta a una comunidad internacional que se ha quedado corta en cuanto a proveer solidaridad internacional y protección a los sirios desplazados". ${ }^{83}$ Según las autoras, ante la ausencia de un mecanismo de asistencia internacional integral y de un programa de reasentamiento, combinado con el bajo número de refugiados que están aceptando los países desarrollados, la mayoría de los refugiados quedarían bajo la responsabilidad y protección de Turquía.

De manera complementaria, el Acuerdo de Readmisión, negociado entre Turquía y la UE en marzo de 2016, que autoriza la deportación a Turquía de refugiados ("migrantes ilegales", según el texto del Acuerdo) que lleguen a Grecia desde ese país y el compromiso de admitir una cantidad igual de refugiados sirios desde Turquía que hayan seguido el procedimiento legal adecuado, podría convertir a Turquía en una zona de amortiguación (o contención) para el ingreso de refugiados a la UE. Esto merece un análisis crítico de la manera en que el derecho puede ser, y es usado, para legalizar la incertidumbre para responder a necesidades políticas coyunturales.

Asimismo, esta crisis humanitaria también se presenta como una oportunidad para que Turquía pueda avanzar hacia un marco jurídico más universal en consonancia con el derecho internacional de refugiados y abrir el debate sobre los desafíos internos del país. Turquía, con sus dilemas y contradicciones, ha asumido una tarea hercúlea ante la indiferencia de otros países de la región y del mundo con muchos más recursos y ha dado un ejemplo de solidaridad.

${ }^{83}$ Yildiz y Uzgören, art. cit., p. 200. 
Şenay Özden plantea un elemento clave, es decir que ninguna "política interna puede quedar en lo interno. Esto es lo que Siria ha hecho al mundo. Lo que está pasando en Turquía ahora no puede quedar en lo interno. Lo mismo va para Europa". ${ }^{84}$ Es absolutamente necesario tener un debate global serio y realista para enfrentar el desafío de la migración, pero fundamentalmente para tratar y resolver las dinámicas que la propician (inequidad social, conflictos armados, cambio climático, violencia criminal).

El Medio Oriente es el actual escenario de un enfrentamiento entre lógicas de poder que combinan viejas prácticas y nuevas tecnologías, en que todos los actores están empujando los límites del derecho internacional y del más básico sentido de humanidad ante la mirada pasiva del mundo. No se trata sólo de gestionar la crisis de los refugiados, sino de resolver un conflicto que ha desangrado un país, ha convulsionado una región y ha golpeado los cimientos morales y políticos al interior de Europa.

Debemos tener claro que la admonición moralista y el levantamiento de muros no es, a la larga, la alternativa viable. Tal vez solucione la coyuntura del fantasma fascista en Europa y coadyuve al proyecto turco de hegemonía regional, pero con el tiempo podría reforzar la deriva autoritaria de la región, profundizar la lucha hegemónica y poner en peligro las raíces mismas de la cohesión social en una zona clave para la estabilidad global.

Bibliografía

AGNUR: Alto Comisionado de Naciones Unidas para los Refugiados, "Syria Regional Refugee Response. Inter-agency Information Sharing Portal", en http://data.unhcr.org/syrianrefugees/ regional.php

${ }^{84}$ Entrevista cit., p. 42. 
ACNUR: Alto Comisionado de Naciones Unidas para los Refugiados, "UnHCR Turkey's Monthly Statistics", en https://data2. unhcr.org/fr/documents/download/57101

Baban, Feyzi, Suzan Ilcan y Kim Rygiel, "Playing Border Politics with Urban Syrian Refugees: Legal Ambiguities, Insecurities, and Humanitarian Assistance in Turkey", Movements: Journal of Critical Migration and Border Regimes Studies, Múnich, vol. 3, núm. 2, 2017, pp. 81-102.

Biehl, Kristen S., "Governing through Uncertainty: Experiences of Being a Refugee in Turkey as a Country for Temporary Asylum", Social Analysis, vol. 59, núm. 1, 2015, pp. 57-75 [Puede verse en http:/ /www.berghahnjournals.com/abstract/journals/socialanalysis/59/1/sa590104.xml, consultado el 4.II.2017].

Çorabatir, Metin, The Evolving Approach to Refugee Protection in Turkey: Assessing Practical and Political Needs, Washington, DC, Migration Policy Institute, 2016.

Daniş, Didem, "A New Period in Turkish Migration Policy: Syrian Refugees as a Foreign Policy Instrument", Saha. Citizen's Assembly-Turkey, núm. esp. 1, 2016, pp. 24-29.

DGMM: Dirección General de Manejo de la Migración, "Temporary Protection Statistics", en http://www.goc.gov.tr/icerik6/temporary-protection_915_1024_4748_icerik 2015.

Durukan, Oktay, "Who are the Refugees, What do They Teach Us?", Saha. Citizen's Assembly Turkey, núm. esp. 1, 2016, pp. 30-36 [Entrevista al autor por Ayşe Çavdar].

Elitok, Seçil Paçaci y Thomas Straubhaar, "Migration from Turkey to Europe: Betwixt and Between?" en Europe, Turkey and the Mediterranean Fostering Cooperation and Strengthening Relations. Europe in Dialogue, Berlín, Bertelsmann Stiftung, Gütersloh, 2012, pp. 97-106.

Erdoğan, Murat, Syrians in Turkey: Social Acceptance and Integration Research, Hacettepe University Migration and Politics Research Centre (HUGO), noviembre de 2014, en https://mmuraterdogan.files.wordpress.com/2014/12/hugo-report-syrians-in-tur- 
key-social-acceptance-and-integration-november-2014-0412 2014-en1.pdf

-, Urban Refugees from "Detachment" to "Harmonization". Siryan Refugees and Process Management of Municipalities: The Case of Istanbul, Estambul, Marmara Belediyeler Birliği, 2017.

y Can Ünver, Perspectives, Expectations and Suggestions of the Turkish Business Sector: Syrians in Turkey, Ankara, Turkish Confederation of Employer Associations, 2015.

Heck, Gerda y Sabine Hess, "Tracing the Effects of the EU-Turkey Deal: The Momentum of the Multi-layered Turkish Border Regime”, Movements: Journal of Critical Migration and Border Regimes Studies, Múnich, vol. 3, núm. 2, 2017, pp. 35-56.

Hudson, John, "U.N. Envoy Revises Syria Death Toll to 400,000", Foreign Policy, 22 de abril de 2016, en http:/ / foreignpolicy.com/ 2016/04/22/u-n-envoy-revises-syria-death-toll-to-400000/

İçduygu, Ahmet y E. Fuat Keyman, "Globalization, Security, and Migration: The Case of Turkey”, Global Governance, vol. 6, núm. 3, 2000, pp. 383-398.

y Evin Millet, "Syrian Refugees in Turkey: Insecure Lives in an Environment of Pseudo-Integration" (Working paper, 13), Roma, Istituto Affari Internazionali, Istanbul Policy Center y Mercator Foundation, 2016.

İneli Ciğer, Meltem, "Implications of the New Turkish Law on Foreigners and International Protection and Regulation no. 29153 on Temporary Protection for Syrians Seeking Protection in Turkey", Oxford Monitor of Forced Migration, vol. 4, núm. 2, 2014, en http:/ / oxmofm.com/vol-4-no-2/

Kirişci, Kemal, “"To Lift or Not to Lift» the Geographical Limitation to the 1951 Geneva Convention Relating to the Status of Refugees: Turkey's Pre-Accession to the eu and Asylum", borrador elaborado para la 4th METU International Relations, Ankara, 30 de junio-2 de Julio de 2005, en http://edam.org. tr/document/Kirisci2.pdf

, Syrian Refugees and Turkey's Challenges: Going Beyond Hospitality. Washington, D.C.: The Brookings Institution, 2014, en https:/ / www.brookings.edu/wp-content/uploads/2016/06/Syrian-Refugees-and-Turkeys-Challenges-May-14-2014.pdf 
Korkut, Umut, "Pragmatism, Moral Responsibility or Policy Change: The Syrian Refugee Crisis and Selective Humanitarianism in the Turkish Refugee Regime", Comparative Migration Studies, vol. 4, núm. 2, 2016, en https:/ / comparativemigrationstudies. springeropen.com/articles/10.1186/s40878-015-0020-9, consultado el 12.V.2017.

Latif, Dilek, "Refugee Policy of the Turkish Republic", The Turkish Yearbook of International Relations, vol. 33, 2000, pp. 1-29.

Mango, Andrew, Atatürk, Nueva York, The Overlook Press, 2002.

Mülteci-Der: Mültecilerle Dayanişma Derneği, Mülteci-Der's Observations on Refugee Situation in Turkey, Izmir, 13 de mayo de 2016, en http://www.multeci.org.tr/en/haberler/multeci-ders-observations-on-refugee-situation-in-turkey

Önerand, N. Asli Şirin y Deniz Genç, "Continuity or Change in Turkey's Mass Migration Policy: From 1989 émigrés to Syrian «guests»", en Guven Seker, Ali Tilbe, Mustafa Okmen, Pınar Yazgan, Deniz Eroglu, Ibrahim Sirkeci (eds.), Turkish Migration Conference 2015, Selected Proceedings, Londres, Transnational Press London, pp. 23-38.

Özden, Şenay, "Syrian Refugees in Turkey”, MPC Research Report 2013/05, Badia Fiesolana, Migration Policy Center (MPC)-European University Institute-Robert Schuman Center for Advanced Studies, 2013.

, "The Opposition Failed Bitterly in the Refugee Issue", Saha. Citizen's Assembly Turkey, núm. esp. 1, 2016, pp. 37-42 [Entrevista al autor por Ayşe Çavdar].

Refugee Rights Turkey, "Asylum Information Database. Country Report: Turkey. European Council of Refugees and Exiles”, 2015, en http:/ / www.asylumineurope.org/reports/country/turkey, consultado el 10.II.2017.

Refugee Studies Center, "The Syrian Humanitarian Disaster. Disparities in Perceptions, Aspirations and Behaviour in Lebanon, Jordan and Turkey", Rsc Research in Brief 3, University of Oxford, diciembre de 2015.

Sari, Elif y Cemile Gizem Dinçer, "Toward a New Asylum Regime in Turkey?”, Movements: Journal of Critical Migration and Border Regimes Studies, vol. 3, núm. 2, 2017, pp. 59-79. 
Singer, Thea, "3Qs: What using the name «Daesh», rather than «ISIS» or «ISIL» Really Means", News@Northeastern, en http:/ / news.northeastern.edu/2015/11/3qs-what-using-the-namedaesh-rather-than-isis-or-isil-really-means

Soykan, Cavidan, "The New Draft Law on Foreigners and International Protection in Turkey", Oxford Monitor of Forced Migration, vol. 2, núm. 2, 2012, pp. 38-47.

The World Bank, “Turkey's Response to the Syrian Refugee Crisis and the Road Ahead", en www.worldbank.org

Yildiz, Ayselin y Elif Uzgören, "Limits to Temporary Protection: Non-Camp Syrian Refugees in İzmir, Turkey", Southeast European and Black Sea Studies, vol. 16, núm. 2, 2006, pp. 195-211, en http://www.tandfonline.com/doi/abs/10.1080/14683857.20 16.1165492?journalCode=fbss20 